\title{
A New Combined Protocol to Treat the Dentin Hypersensitivity Associated with Non-Carious Cervical Lesions: A Randomized Controlled Trial
}

\author{
Felice Femiano ${ }^{1}$, Rossella Femiano ${ }^{1}$, Luigi Femiano ${ }^{1}$, Ludovica Nucci ${ }^{1}$, Giuseppe Minervini ${ }^{1, * \mathbb{D} \text {, }}$ \\ Alessandro Antonelli ${ }^{2}\left(\mathbb{D}\right.$, Francesco Bennardo ${ }^{2} \mathbb{D}$, Selene Barone ${ }^{2} \mathbb{D}$, Nicola Scotti ${ }^{3}$, Vittoria Sorice ${ }^{4}$ \\ and Rossella Sorice ${ }^{1}$
}

1 Multidisciplinary Department of Medical-Surgical and Dental Specialties, University of Study of Campania, Luigi Vanvitelli, Via De Crecchio 6, 83138 Naples, Italy; femiano@libero.it (F.F.); rossella.femiano@libero.it (R.F.); 1_femiano@hotmail.it (L.F.); ludortho@gmail.com (L.N.); rossella.sorice@studenti.unicampania.it (R.S.)

2 Department of Health Sciences, School of Dentistry, Magna Græcia University of Catanzaro, 88100 Catanzaro, Italy; antonellicz@gmail.com (A.A.); fbennardo92@gmail.com (F.B.); barone.selene19@gmail.com (S.B.)

3 Department of Surgical Sciences, University of Turin, 10125 Torino, Italy; nicola.scotti@unito.it

4 Chesterfield Royal Hospital Foundation Trust, University of Derby, Kedleston Rd., Derby DE22 1 GB, UK; vittoria.sorice@nhs.net

* Correspondence: minervinigiuseppe@hotmail.it; Tel.: +39-3289129558

\section{check for}

updates

Citation: Femiano, F.; Femiano, R.; Femiano, L.; Nucci, L.; Minervini, G.; Antonelli, A.; Bennardo, F.; Barone, S.; Scotti, N.; Sorice, V.; Sorice, R.; et al. A New Combined Protocol to Treat the Dentin Hypersensitivity Associated with Non-Carious Cervical Lesions: A Randomized Controlled Trial. Appl. Sci. 2021, 11, 187. https://dx.doi.org/10.3390/app 11010187

Received: 17 November 2020 Accepted: 24 December 2020 Published: 28 December 2020

Publisher's Note: MDPI stays neutral with regard to jurisdictional claims in published maps and institutional affiliations.

Copyright: (c) 2020 by the authors. Licensee MDPI, Basel, Switzerland. This article is an open access article distributed under the terms and conditions of the Creative Commons Attribution (CC BY) license (https: / / creativecommons.org/ licenses/by/4.0/).

\begin{abstract}
The goal of this research is to assess the desensitizing effect of a diode laser on noncarious cervical lesions (NCCLs) responsible for dentin hypersensitivity $(\mathrm{DH})$ in two separate output forces implemented both independently and in sequential combination modalities. A randomized controlled trial for this study was applied. Sixty-nine NCCLs responsible for DH pain with severity between 6 and 9 on the VAS scale were considered. Three study groups were developed using just one lesion from three different quadrants of the oral cavity of each patient. All treatment procedures were conducted using a laser diode $(810 \mathrm{~nm}, 5 \mathrm{~W})$ with varying power outputs used separately or in combination. The pain by DH was evaluated at baseline, at treatment completion, and at 15 days and 3 months after each laser procedure. Data analysis was performed using a Wilcoxon test for paired samples, a one-way ANOVA test, and an unpaired t-test. The significant reduction of the mean VAS score was estimated in each study group immediately and at 15 days and 3 months after the end of treatment and compared with the baseline mean VAS score ( $p$-value $<0.0001)$. The best result concerning the improvement of DH symptomatology was assessed when a combined protocol of two different output powers of the diode laser was used. The authors conclude that the diode laser $(810 \mathrm{~nm})$ therapy procedure combining two separate output forces (low and high power) can improve the painful symptoms of DH from NCCLs.
\end{abstract}

Keywords: dentin hypersensitivity; non-carious cervical lesions; diode laser; LLLT; high-power laser therapy

\section{Introduction}

An international workshop describes Dentin Hypersensitivity (DH) as follows: “Dentin Hypersensitivity is characterized by short, sharp pain arising from exposed dentin in response to stimuli, typically thermal, evaporative, tactile, osmotic or chemical and which cannot be ascribed to any other dental defect or pathology" [1]. DH frequently affects individuals aged 30 to 40 years old and is often associated with non-carious cervical lesions (NCCLs) [2-4]. It occurs when dentinal tubules are exposed to the oral environment, and this is well explained by the hydrodynamic theory of Brännström [5]. This theory suggests that, when one of these stimuli are applied to the fluid within the patent dentinal tubules, the resulting pressure changes activate the mechanical receptors causing the 
transmission of the stimulus to the pulpal nerves $[5,6]$. The severity of symptoms does not depend on the breadth and depth of lesions, but on the number of non-occluded tubules exposed to the oral cavity, consequently, the sealing of exposed and patent tubules should reduce or eliminate the pain and discomfort of the patient. Several treatments belonging to the tubule-occluding agent family [7] have been proposed including protein binding and calcium compounds deposition within tubules with casein phosphopeptide [8,9], peritubular mineral deposition, and sodium fluoride $(\mathrm{NaF})$. Sometimes, these treatments, as well as the use of nerve depolarization therapies (topical potassium nitrate), could be ineffective or might produce short-lived desensitizing effects [10]. However, a recent systematic review comparing DH treatments on the based on its efficacy and effect duration confirms the following: for an immediate (until 7 days) DH reduction, the use of glutaraldehyde with HEMA, glass ionomer cement, and a laser is significant; the use of stannous fluoride, glutaraldehyde with HEMA, hydroxyapatite, glass ionomer cement, and a laser provides a medium-term (until 1 month) DH decrease; for a more long-term DH reduction, the use of potassium nitrate, arginine, glutaraldehyde with HEMA, hydroxyapatite, adhesive systems, glass ionomer cement, and a laser is significant. Overall, the review shows the efficacy of all active ingredients in reducing $\mathrm{DH}$ at different time points, but the treatments must be completely in-office to achieve an immediate and persisting efficient DH decrease [11]. In a meta-analysis by $\mathrm{Hu} \mathrm{ML}$ et al., all types of lasers showed a greater desensitizing effect on DH than the negative controls, both in the immediate and long term [12]. Regrettably, there are conflicting pieces of evidence amongst the authors on which laser achieves the best outcome for the treatment of DH. In fact, Yilmaz et al. (2011) [13] reported an equal desensitizing effect for a diode laser and Er,Cr:YSGG lasers, while Dilsiz et al. (2009) [14] stated that an Nd:YAG laser is more efficient than a diode laser. In a recent study by Pourshahidi S. et al. (2019), a split-mouth randomized clinical study compared Er, Cr:YSGG and diode laser effects on DH using a pulse width of 140-200 $\mu$ s. The study findings showed that the DH reduction after an Er,Cr:YSGG laser application was considerably higher than that after a diode laser application at a 1-month postoperative interval [15].

It seems that a diode laser with different output powers range (low and high) could act on DH by two different mechanisms: high-power lasers could cause a melt of the external surface peritubular dentin, while low-power lasers could induce anti-inflammatory effects with an increase in the metabolic activity of odontoblasts causing the production of new dentin [14].

The aim of this study was to evaluate the effectiveness of a diode laser on pain caused by DH associated with NCCLs by comparing three different laser therapy protocols: high and low power used individually and a combined method using first a low- and then a high-power laser.

\section{Materials and Methods}

A split-mouth study design was used to allocate patients to three groups: one standard care group using Low-Level Laser Therapy (LLLT), one standard care group using HighPower Laser Therapy (HPLT), and one intervention group using a new, combined diode laser therapy, Low-High combined Laser Therapy (LHLT). The randomization used was in the ratio 1:1:1.

For this research, 4 operators, 3 experienced clinicians, and a non-clinical researcher, were recruited to avoid bias in carrying out the study and in the interpretation of the results.

The three clinicians were recruited to perform the following functions: an enrollment of subjects affected by DH related to NCCLs, an evaluation of pain intensity per lesion after the evaporative test (ET), and the administration of the treatments in analysis. The researcher was trained to perform randomization to determine which NCCLs to treat with a diode laser. Lastly, the operator responsible for the enrollment of the subjects also carried out the analysis of the ET data. 


\subsection{Selection of Lesions}

The vistibular surfaces of the teeth of 172 subjects ( 88 females and 84 males aged 24 to 70 years old), arrived from January 2017 to July 2019 at the conservative outpatient department of the University of Campania "Luigi Vanvitelli," were examined by the first operator using a probe tip and a $x 4$ magnifying lens. The tip of the probe was held perpendicularly to the tooth surface and inserted to the bottom of the gingival sulcus crossing the cement-enamel junction (CEJ). Any irregularity in the cervical enamel and at the CEJ of the teeth was considered as NCCLs. All subjects signed an Informed Consent form after verbal and written information on the study was provided.

The procedures of this study were in accordance with the institutional and national ethical standards on human experimentation and the Helsinki Declaration of 1975, as revised in 2000, and they were approved by the Ethics Committee of AORN of Ospedali dei Colli with N. 1374.

The first operator selected 87 adult patients ( 47 females and 40 males aged 24 to 70 years old) who complained of dentin hypersensitivity symptoms related to NCCLs.

A total of 372 NCCLs were screened on the vestibular surface of the tooth (Figure 1). The NCCLs excluded from the analysis presented the following: (1) an association of the NCCLs with periodontal pockets or gingival recession; (2) an additional presence of carious lesions on the affected tooth or a pre-existing restoration; (3) evidence of cracks; (4) previous endodontic treatment; (5) primary teeth and subgingival NCCLs.

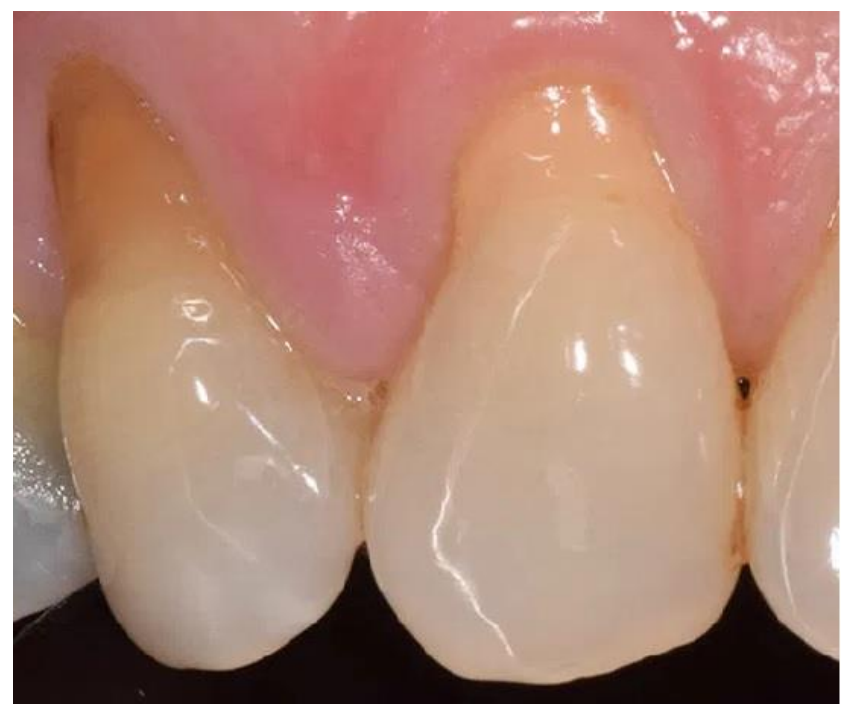

Figure 1. A non-carious cervical lesion (NCCL).

The second researcher was employed to classify the NCCLs to be enrolled in this analysis on the basis of the pain sensation severity elicited by each NCCL using ETs: baseline (t0). A visual analog scale (VAS) was used to assess the severity of pain, enabling patients to record their perceived level of pain from 0 (the best possible pain status) to 10 (the worst possible pain status).

Only NNCLs associated with DH scoring 6-9 on the VAS after an ET were included in this study.

Three study groups were established by using only one lesion per patient in 3 different quadrants of the oral cavity to obtain 3 lesions per patient to be treated with individual different therapeutic protocols. Blinded to the protocol selection required for each tooth treated, the second investigator was also employed to examine the intensity of pain sensation elicited by each NCCL by an ET according to the following timeline: at treatment completion ( $\mathrm{t} 1)$, at 15 days ( $\mathrm{t} 2)$, and at 3 months ( $\mathrm{t} 3$ ). 
Allocation to the specific study group for each NCCL was achieved by the third operator using a randomization software, i.e., random allocation software [16].

Moreover, all patients with NCCLs associated with DH underwent anamnestic analysis with the intention of discovering and eliminating the causal factors. Instructions were provided on the adequate management of oral hygiene to carry out at home and proper dietary requirements.

The selected teeth with NCCLs were flossed and polished before the administration of the treatment, and cotton rolls were used for isolation.

\subsection{Evaporative Test (ET)}

The degree of tooth sensitivity was determined by an air stimulus defined as a $3 \mathrm{~s}$ cold air blast (temperature range of $19-20^{\circ} \mathrm{C}$ ) at a distance of $2-3 \mathrm{~mm}$ from the test site. The tooth under examination was isolated using cotton rolls and by shielding the neighboring teeth with the gloved fingers of the operator. All patients were asked to report their degree of pain for each NCCL after the ET using the VAS scale at each point of the timeline previously described.

\subsection{Laser Treatment Session}

The fourth operator carried out all treatment protocols using the fiber of a $400 \mu \mathrm{m}$ diode laser (Soft Touch, Creation Medical Laser; $810 \mathrm{~nm}, 5 \mathrm{~W}$ ) with a distance of 1-2 mm from the dental surface and with vertical and horizontal movements to cover the entire surface of the NCCL of each tooth evaluated in order to avoid the production of thermal effects within the tooth. Three different laser treatments (LTs) were used: low and high output powers used individually and a combined method using first the low- and then the high-power laser.

The set parameters of each LT for the study groups were as follows:

Low-Level Laser Therapy (LLLT): $0.2 \mathrm{~W}$ in continuous emission (CW) for $60 \mathrm{~s}$ and treatment repeated after $24 \mathrm{~h}$; High-Power Laser Therapy (HPLT): $2 \mathrm{~W}$ in pulse mode for $60 \mathrm{~s}$ and treatment repeated after $24 \mathrm{~h}$; Low-High combined Laser Therapy (LHLT): NCCLs were treated with combined diode laser therapy. In detail, the same lesion was exposed to the first stage of laser treatment following the LLLT protocol. After $24 \mathrm{~h}$, the same lesion underwent the second session of irradiation applying the HPLT protocol (Table 1). A gap of $24 \mathrm{~h}$ between the irradiating sessions was used to allow the results of the first session to consolidate.

Table 1. Laser parameters used for each study group. LLLT: Low-Level Laser Therapy; HPLT: High-Power Laser Therapy; LHLT: Low-High combined Laser Therapy; CW: Continuous Wave.

\begin{tabular}{cccccc}
\hline & Fiber & Wattage & Emission & First Stage & After 24 h \\
\hline LLLT & $400 \mu \mathrm{m}$ & 0.2 & $\mathrm{CW}$ & $60 \mathrm{~s}$ & $60 \mathrm{~s}$ \\
\hline HPLT & $400 \mu \mathrm{m}$ & 2 & Pulse mode & $60 \mathrm{~s}$ & $60 \mathrm{~s}$ \\
\hline \multirow{2}{*}{ LHLT } & $400 \mu \mathrm{m}$ & 0.2 & CW & $60 \mathrm{~s}$ & \\
& $400 \mu \mathrm{m}$ & 2 & Pulse mode & & $60 \mathrm{~s}$ \\
\hline
\end{tabular}

In Figure 2, a flow chart of the study project was shown.

\subsection{Statistical Analysis}

The mean and the standard error of the VAS score at each time point $(\mathrm{t} 0, \mathrm{t} 1, \mathrm{t} 2$, and $\mathrm{t} 3)$ were estimated for each study group. Data analysis was conducted using the Wilcoxon test for paired samples $[17,18]$ to assess for significant differences among the averages observed at $\mathrm{t} 1, \mathrm{t} 2$, and $\mathrm{t} 3$ compared to $\mathrm{t} 0 \mathrm{in}$ each study group. The mean of VAS scores obtained at $\mathrm{t} 0$ for each patient was compared among the three study groups applying a one-way ANOVA test. An unpaired t-test was applied to compare the averages of VAS scores for the pairs: LLLT vs. HPLT, LLLT vs. LHLT, and HPLT vs. LHLT. 


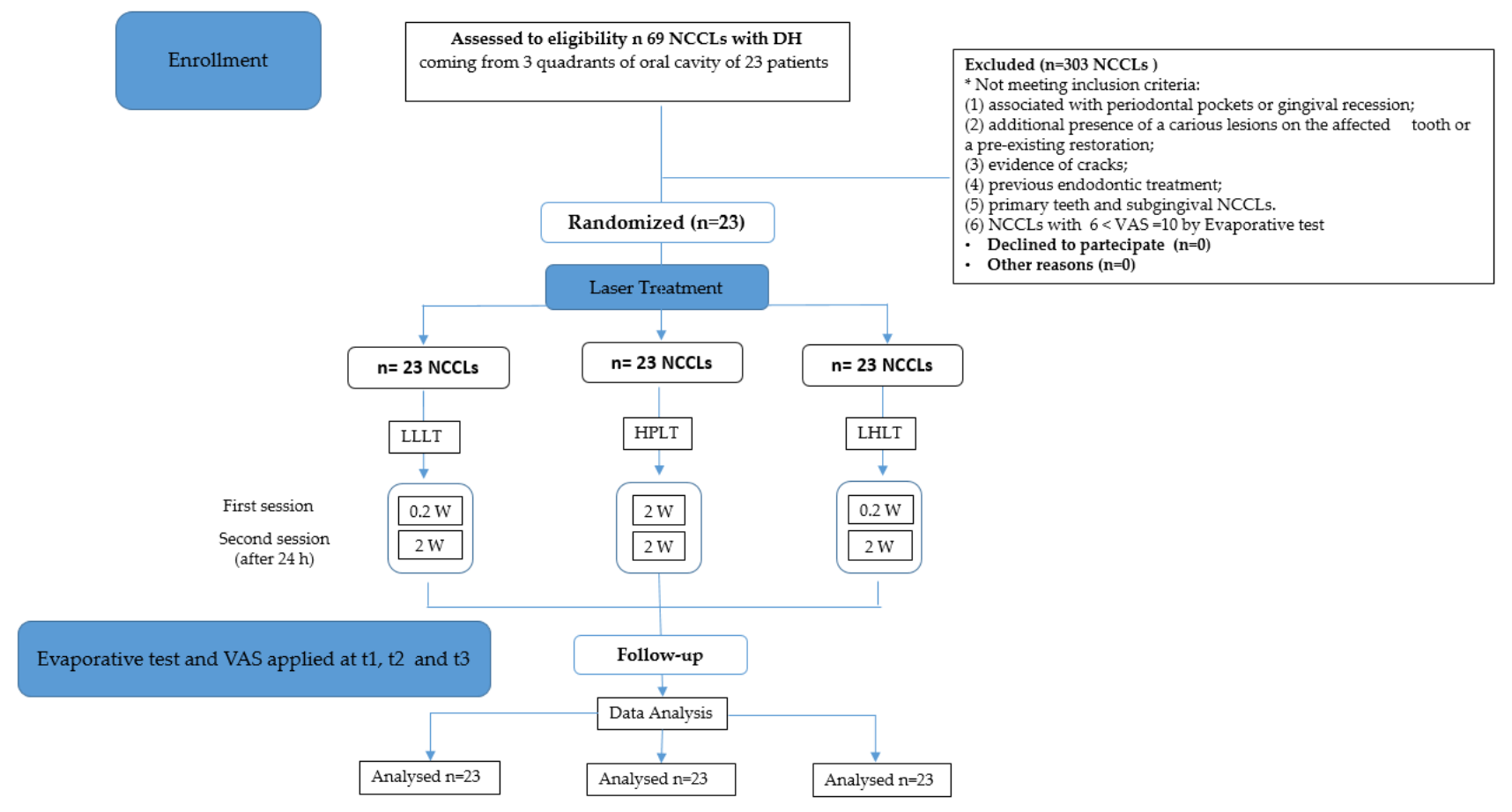

Figure 2. Flow diagram showing the study project.

All the statistics used for the data analysis were implemented in GraphPad Prism V 6.0. Values of $p<0.05$ were accepted as statistically significant.

Moreover, in each study group, the mean residual pain (Pi) was estimated evaluating the mean VAS score at $\mathrm{t} 1, \mathrm{t} 2$, and $\mathrm{t} 3$ times against the mean VAS scores of $\mathrm{t} 0$. The Equation applied was

$$
\text { for } \mathrm{i}=0,1,2,3 \frac{(\text { Mean } \cdot \text { VAS } \cdot \text { score }) t_{i}}{(\text { Mean } \cdot \text { VAS } \cdot \text { score }) t_{0}} \cdot X \cdot 100
$$

The improvement of DH symptomology was then obtained as the difference between 100 , the best possible result, and the mean residual pain $\left(P_{i}\right)$ estimated at $t 1, t 2$, and $t 3$. The formula applied was

$$
\text { for } \mathrm{i}=0,1,2,3 \mathrm{I}_{\mathrm{i}}=100-\mathrm{P}_{\mathrm{i}}
$$

A sample consisting of 90 NCCLs with DH needed a power of $80 \%$ to demonstrate an effect size of $30 \%$ with a Wilcoxcon test for paired samples and with a first type error of 0.05 .

\section{Results}

The final study sample used for our analysis included 69 NCCLs associated with $\mathrm{DH}$ and belonging to 23 patients, 15 of which were females and 8 of which males, with a mean age of 41.4 years. All patients completed the study. The NCCLs selected for analysis included those that did not meet the exclusion criteria, reported in the Material and Methods section, and that showed a VAS score between 6 and 9 in three different quadrants of the mouth for each patient. Finally, thanks to randomization software, the 69 NCCLs were assigned to the three study groups, each consisting of 23 NCCLs and treated with a different laser therapy. In the same patient, one lesion per selected quadrant was included in a different study group.

The values of mean and standard error of the VAS scores for each study group are shown in Table 2 for each time point of the therapeutic protocol: baseline $(\mathrm{t} 0)$, at treatment completion (t1), at 15 days (t2), and at 3 months (t3). 
Table 2. The mean and the standard error of the VAS score are shown at each time point ( $\mathrm{t} 0, \mathrm{t} 1$, t2, and t3) for each study group. LLLT: Low-Level Laser Therapy; HPLT: High-Power Laser Therapy; LHLT: Low-High combined Laser Therapy; CW: Continuous Wave ${ }^{*}=p$-value of Wilcoxon test $<0.0001$, comparison performed with the mean VAS score at $\mathrm{t} 0$. The bold text shows the significant $p$-value obtained with the Wilcoxon text by comparison of the mean VAS score between $\mathrm{t} 1$ and $\mathrm{t} 2$ and between $\mathrm{t} 2$ and $\mathrm{t} 3$.

\begin{tabular}{ccccc}
\hline Study Groups & t0 & t1 & t2 & t3 \\
\hline$L L L T$ & $7.04 \pm 0.20$ & $3.57 \pm 0.27^{*}$ & $3.83 \pm 0.21^{*}$ & $4.39 \pm 0.23^{*}$ \\
$H P L T$ & $7.04 \pm 0.20$ & $2.87 \pm 0.17^{*}$ & $4.26 \pm 0.16^{*}$ & $4.65 \pm 0.17^{*}$ \\
$L H L T$ & $7.09 \pm 0.20$ & $2.83 \pm 0.15^{*}$ & $3.09 \pm 0.15^{*}$ & $3.52 \pm 0.12^{*}$ \\
\hline
\end{tabular}

A significant reduction of the mean VAS score was estimated for each study group at $\mathrm{t} 1, \mathrm{t} 2$, and $\mathrm{t} 3$ compared with the mean VAS score assessed at $\mathrm{t} 0$ ( $p$-value of Wilcoxon test $<0.0001$ for each tested pair and for each study group).

No significant difference in the mean of VAS scores at 0 was appraised among the three study groups ( $p$-value of the one-way ANOVA test: 0.9846 ; mean values are shown in Table 2).

The comparison of the means of the VAS scores at $\mathrm{t} 1$, $\mathrm{t} 2$, and $\mathrm{t} 3$ between each pair of study groups reveals the following: a statistically significant difference for LLLT vs. LHLT at each tested point of the laser treatment timeline; for the HPLT vs. LHLT comparison, the difference is extremely significant only at $\mathrm{t} 2$ and $\mathrm{t} 3$; for LLLT vs. HPLT, a significant difference was observed only at $\mathrm{t} 1$ (the $p$-values are shown in Table 3 ).

Table 3. Comparison of the mean values of VAS estimated at $\mathrm{t} 1, \mathrm{t} 2$, and $\mathrm{t} 3$ for the three pairs of study groups: LLLT vs. HPLT, LLLT vs. LHLT, and HPLT vs. LHLT. ${ }^{*}=p$-value $<0.05 ;{ }^{* * *}=p$-value $<0.005$. LLLT: Low-Level Laser Therapy; HPLT: High-Power Laser Therapy; LHLT: Low-High combined Laser Therapy; CW: Continuous Wave.

\begin{tabular}{cccc}
\hline Study Groups & $\mathbf{t 1}$ & $\mathbf{t} 2$ & $\mathbf{t}$ \\
\hline LLLT vs. HPLT & $0.0326^{*}$ & 0.0993 & 0.3740 \\
LLLT vs. LHLT & $0.0194^{*}$ & $0.0060^{*}$ & $0.0019^{* * *}$ \\
HPLT vs. LHLT & 0.8485 & $0.0001^{* * *}$ & $0.0001^{* * *}$ \\
\hline
\end{tabular}

An improvement of DH symptomology was registered for all study groups. The HPLT and LHLT present the best results, $59 \%$ and $60 \%$, respectively, immediately after the laser treatment (t1), while the improvement of DH was $49 \%$ for LLLT. On the other hand, LLLT and LHLT reveal a similar trend in the reduction of improvements, which was more gradual over time than the HPLT, which showed a rapid decrease at $\mathrm{t} 2$ ( $59 \%$ at $\mathrm{t} 1 \mathrm{vs.} 39 \%$ at $\mathrm{t} 2$ ), but shows unvaried values from $\mathrm{t} 2$ to $\mathrm{t} 3$ (Table 2).

\section{Discussion}

In the literature, the ability of different types of lasers in sealing patent dentinal tubules has been evaluated, but studies carried out on which laser achieves the best outcome for the treatment of DH showed discordant evidence, which could be due to several factors such as the types of lasers, fibers, power output, exposure time, and radiation frequency [19]. Several studies have shown the efficiency of a diode laser [20-23] and of its different wavelengths $(780,900$, and $810 \mathrm{~nm})$ in the treatment of $\mathrm{DH}$ [24]. A diode laser of $810 \mathrm{~nm}$ is a high-energy laser with thermal effects that develop in-depth by virtue of its physiological target. However, by acting on the energy powers of the laser and on the mode of use, the thermal effects can be limited at the surface layers of hard tissues when we use the laser with an energy power of $2 \mathrm{~W}$ and in pulsed mode (HPLT) with a non-contact fiber; by using lower powers, $0.2 \mathrm{~W}$, with a non-contact fiber and in CW mode (LLLT), thermal effects can be avoided, and photo-stimulating activities for the tissues can be promoted instead. 
The rationale behind the use of two different output powers of the diode laser is based on the different interaction that each intensity of a laser has with the tissues: a photothermal effect with the thermodynamic behaviour of tissue water content and bio-stimulant effects on dental tissues. In the present study, we have appraised the effectiveness of three different treatments based on the use of a diode laser (LLLT, HPLT, and LHLT), focusing on the different percentage of pain reduction and on the persistence of the effect over time.

Our results underline that more immediate improvements, obtained with a singleoutput power, are not long-lasting, while others that can be achieved over a longer period of time are more persistent $[14,25]$.

The effectiveness of each laser therapy was gauged by estimating the improvement of DH symptomatology on three different NCCLs by comparing the mean VAS score obtained at the end of treatment ( $\mathrm{t} 1)$, after 15 days ( $\mathrm{t} 2)$, and after 3 months ( $\mathrm{t} 3)$, with the mean VAS values at baseline (t0).

A clear improvement, both in the short and long term, was documented for all the NCCLs in all study groups, with the best results obtained in LHLT. Immediately after the end of treatment (t1), HPLT and LHLT showed the same improvement of DH symptomatology compared to LLLT (59.29\% and $60.12 \%$ vs. $49.38 \%$ ). In fact, no significant difference was estimated between the mean VAS score of HPLT and LHLT at t1, while both averages were statistically different from the mean VAS score of LLLT for the same time point. After 15 days and after three months, the effectiveness of LHLT was still greater (56.44\% and 50.62\%, respectively) than the laser treatments based on the use of singleoutput power, applied in LLLT and HPLT, respectively. This result was supported by the fact that the averages of VAS scores of LLLT vs. LHLT and of HPLT vs. LHLT were statistically different.

In HPLT, the lasers with high power can improve the symptomatology of DH mainly by the photothermal effect that melts the hard tissue on the surface with the smear layer occluding the tubules. In this way, the laser blocks the movement of the fluids in the dentinal tubules, which is the fundament of the hydrodynamic theory of DH pain. The studies of Lopes AO and Aranha ACC (2013) demonstrate that the sealing of dentinal tubules, obtained with the use of a high-power laser, represents an effective treatment strategy in reducing DH [26]. A photo-thermal effect was also reported by Liu et al. for the Nd:YAG laser, which allows a sealing depth of approximately 4 microns due to the melting of dentin and the closure of exposed dentinal tubules [26]. The short-term efficacy of a high-power laser can be precisely correlated to the rapidity with which the physical-thermal effect closes the patent dentinal tubules, but this haste can also cause early relapse and therapy failure if the causal factors persist (i.e., mechanical abrasion or microbial action), which could induce a possible loss of the superficial layer of dentin [20,27].

In LLLT, the desensitizing action of the low-power laser on DH was compared with the action of desensitizing paste containing $8 \%$ arginine-calcium carbonate by Bal et al. (2015) [28]. The efficiency of the low-power laser seems to be greater when lasers with high-tissue penetration are used, such as the $810 \mathrm{~nm}$ diode laser and Nd:YAG. Whitters et al. (1995) and Femiano et al. (2018) verified the analgesic effect of low power using either an $\mathrm{Nd}$ :YAG laser $(1.1 \mathrm{~W})$ or a diode laser $(0.1 \mathrm{~W})$ during routine dental restoration procedures for carious lesions with recovery to baseline pain threshold values after 60 min $[29,30]$. This output power showed an analgesic effect on the pulp tissue due to the possible depolarizing effect of $C$ and $A \delta$ nerve fibers [31] and an anti-inflammatory effect due to the bio-stimulation of the pulp tissue that activated the odontoblasts to counter the production of irregular secondary dentin. The action on the inner layer of dentin with the activation of odontoblasts can explain the slower action of the low-power laser on pain reduction associated with $\mathrm{DH}$ and, at the same time, the longer-lasting effect compared with the action of a high-power laser that is restricted to the external surface of dentin [32].

In LHLT, a combined and definite sequence of two different output powers of diode lasers was used on the NCCLs associated with DH. This approach dictated the use of low power first and subsequently high power in order to have a prompt improvement in pain 
that lasts over time. The rationale for this approach is based on the hypothesis that the mechanical effect, induced by a high-power laser, creates a barrier on the external side of the tubules, which protects the internal environment. In this way, the odontoblasts, already activated by the biological effect and induced by the low-power laser, have a greater chance of organizing the defense on the internal side of the tubules and eliciting the production of secondary dentin. Moreover, the sealing of the external side of dentinal tubules, induced by mechanical action, leads to an immediate improvement of the DH symptomatology. The opposite sequence of the use of output powers should not have the same efficiency in the long term, because, if the external sealing is induced by a high-power laser before bio-stimulation of the internal side of the dentin is achieved, it could create a barrier that prevents the low-power laser from reaching the most inner layer and thus activating the odontoblasts.

\section{Conclusions and Limitations}

The goal of this study is to test the efficacy of diode lasers on pain caused by $\mathrm{DH}$ associated with NCCLs by evaluating three different protocols of laser therapy: high and low power used independently compared to a hybrid procedure using first the low- and then the high-power laser. Our study has shown that the best patient outcome is achieved when combining the bio-stimulation effect of the slower action of the low-power laser on $\mathrm{DH}$-associated pain relief and the immediate pain relief action of the high-power laser on the external sealing.

Taking into account the findings obtained, we suggest a new laser therapy procedure incorporating two separate output powers of the diode laser to treat DH while its symptomatology is evoked in the presence of NCCLs to achieve a more durable regulation of pain.

The authors take into account the study limitations, namely, the small sample of lesions available, the lack of evidence to support this new hybrid protocol, and the timing of the COVID-19 pandemic preventing any further evaluation of the protocols. Thus, further research on this new and promising application is required.

Author Contributions: Data curation, S.B.; Formal analysis, R.F., L.F., and A.A.; Investigation, R.F., L.F., and F.B.; Methodology, G.M. and N.S.; Project administration, S.B.; Software, L.N.; Supervision, N.S. and R.S.; Visualization, L.N.; Writing-Original draft, G.M. and R.S.; Writing-Review \& editing, F.F., R.S., and V.S. All authors have read and agreed to the published version of the manuscript.

Funding: This research received no external funding.

Institutional Review Board Statement: The study was conducted according to the guidelines of the Declaration of Helsinki of 1975, as revised in 2000, and approved by the Ethics Committee of AORN of Ospedali dei Colli with N. 1374 of 21/12/2016

Informed Consent Statement: Informed consent was obtained from all subjects involved in the study. All subjects gave their consent to the publication of data for scientific purposes.

Data Availability Statement: Data Availability Statements are in section "MDPI Research Data Policies" at https: / www.mdpi.com/ethics. All raw data are available for consultation and for a collaboration request at the following email address: femiano@libero.it.

Acknowledgments: The authors thank the Multidisciplinary Department of Medical-Surgical and Dental Specialties, University of Campania "L. Vanvitelli" for the received support through the program VALERE.

Conflicts of Interest: The authors declare no conflict of interest. 


$\begin{array}{ll}\text { Abbreviations } & \\ \text { Non-carious cervical lesions } & \text { NCCLs } \\ \text { Dentin hypersensitivity } & \text { DH } \\ \text { Cement-enamel junction } & \text { CEJ } \\ \text { Visual analogue scale } & \text { VAS } \\ \text { Evaporative Test } & \text { ET } \\ \text { Low-Level Laser Therapy } & \text { LLLT } \\ \text { High-power laser therapy } & \text { HPLT } \\ \text { Low-High combined Laser Therapy } & \text { LHLT }\end{array}$

\section{References}

1. Dowell, P.; Addy, M. Dentine hypersensitivity-A review: Aetiology, symptoms and theories of pain production. J. Clin. Periodontol. 1983, 10, 341-350. [CrossRef] [PubMed]

2. Felix, J.; Ouanounou, A. Dentin Hypersensitivity: Etiology, Diagnosis, and Management. Compend. Contin. Educ. Dent. 2019, 40, 653-658. [PubMed]

3. Moraschini, V.; da Costa, L.S.; Dos Santos, G.O. Effectiveness for dentin hypersensitivity treatment of non-carious cervical lesions: A meta-Analysis. Clin. Oral. Investig. 2018, 22, 617-631. [CrossRef] [PubMed]

4. Clark, D.; Levin, L. Non-surgical management of tooth hypersensitivity. Int. Dent. J. 2016, 66, 249-256. [CrossRef]

5. Brännström, M. Etiology of dentin hypersensitivity. Proc. Finn. Dent. Soc. 1992, 88 (Suppl. S1), 7-13.

6. Zhou, K.; Liu, Q.; Yu, X.; Zeng, X. Laser therapy versus topical desensitising agents in the management of dentine hypersensitivity: A meta-Analysis. Oral. Dis. 2020, 21. [CrossRef]

7. Creeth, J.; Gallob, J.; Sufi, F.; Qaqish, J.; Gomez-Pereira, P.; Budhawant, C.; Goyal, C. Randomised clinical studies investigating immediate and short-term efficacy of an occluding toothpaste in providing dentine hypersensitivity relief. BMC Oral. Health 2019, 4, 98. [CrossRef]

8. Bou Chebel, F.; Zogheib, C.M.; Baba, N.Z.; Corbani, K.A. Clinical Comparative Evaluation of Nd: YAG Laser and a New Varnish Containing Casein Phosphopeptides-Amorphous Calcium Phosphate for the Treatment of Dentin Hypersensitivity: A Prospective Study. J. Prosthodont. 2018, 27, 860-867. [CrossRef]

9. Sgreccia, P.C.; Barbosa, R.E.S.; Damé-Teixeira, N.; Garcia, F.C.P. Low-power laser and potassium oxalate gel in the treatment of cervical dentin hypersensitivity-A randomized clinical trial. Clin. Oral. Investig. 2020, 24, 4463-4473. [CrossRef]

10. Hall, C.; Sufi, F.; Milleman, J.L.; Milleman, K.R. Efficacy of a 3\% potassium nitrate mouth rinse for the relief of dentinal hypersensitivity: An 8-Week randomized controlled study. J. Am. Dent. Assoc. 2019, 150, 204-212. [CrossRef]

11. Marto, C.M.; Baptista Paula, A.; Nunes, T.; Pimenta, M.; Abrantes, A.M.; Pires, A.S.; Laranjo, M.; Coelho, A.; Donato, H.; Botelho, M.F.; et al. Evaluation of the efficacy of dentin hypersensitivity treatments-A systematic review and follow-up analysis. J. Oral. Rehabil. 2019, 46, 952-990. [CrossRef] [PubMed]

12. Hu, M.L.; Zheng, G.; Han, J.M.; Yang, M.; Zhang, Y.D.; Lin, H. Effect of Lasers on Dentine Hypersensitivity: Evidence from a Meta-analysis. J. Evid. Based Dent. Pract. 2019, 201, 115-130. [CrossRef]

13. Yilmaz, H.G.; Kurtulmus-Yilmaz, S.; Cengiz, E.; Bayindir, H.; Aykac, Y. Clinical evaluation of Er,Cr:YSGG and GaAlAs laser therapy for treating dentine hypersensitivity: A randomized controlled clinical trial. J. Dent. 2011, 39, 249-254. [CrossRef] [PubMed]

14. Dilsiz, A.; Canakci, V.; Ozdemir, A.; Kaya, Y. Clinical evaluation of Nd: YAG and 685-nm diode laser therapy for desensitization of teeth with gingival recession. Photomed. Laser Surg. 2009, 27, 843-848. [CrossRef] [PubMed]

15. Pourshahidi, S.; Ebrahimi, H.; Mansourian, A.; Mousavi, Y.; Kharazifard, M. Comparison of Er,Cr:YSGG and diode laser effects on dentin hypersensitivity: A split-mouth randomized clinical trial. Clin. Oral. Investig. 2019, 23, 4051-4058. [CrossRef]

16. Saghaei, M. Random allocation software for parallel group randomized trials. BMC Med. Res. Methodol. 2004, 4, 26. [CrossRef]

17. McGee, M. Case for omitting tied observations in the two-sample t-test and the Wilcoxon-Mann-Whitney Test. PLoS ONE 2018, 13, e0200837. [CrossRef]

18. Santos, A.E.C.G.D.; Bussadori, S.K.; Pinto, M.M.; Pantano Junior, D.A.; Brugnera, A., Jr.; Zanin, F.A.A.; Rodrigues, M.F.S.D.; Motta, L.J.; Horliana, A.C.R.T. Evaluation of in-office tooth whitening treatment with violet LED: Protocol for a randomised controlled clinical trial. BMJ Open 2018, 4, e021414. [CrossRef] [PubMed]

19. Torabinejad, M.; Ravanshad, S.H. Effects of Nd: YAG laser on apical seal of teeth after apicoectomy and retrofill. J. Endod. 1992, 18, 371-375. [CrossRef]

20. Sicilia, A.; Cuesta-Frechoso, S.; Suárez, A.; Angulo, J.; Pordomingo, A.; De Juan, P. Immediate efficacy of diode laser application in the treatment of dentine hypersensitivity in periodontal maintenance patients: A randomized clinical trial. J. Clin. Periodontol. 2009, 36, 650-660. [CrossRef]

21. Orhan, K.; Aksoy, U.; Can-Karabulut, D.C.; Kalender, A. Low-level laser therapy of dentin hypersensitivity: A short-term clinical trial. Lasers Med. 2011, 26, 591-598. [CrossRef] [PubMed]

22. Raichur, P.S.; Setty, S.B.; Thakur, S.L. Comparative evaluation of diode laser, stannous fluoride gel, and potassium nitrate gel in the treatment of dentinal hypersensitivity. Gen. Dent. 2013, 61, 66-71. [PubMed] 
23. Flecha, O.D.; Azevedo, C.G.S.; Matos, F.R.; Vieira-Barbosa, N.M.; Ramos-Jorge, M.L.; Gonçalves, P.F.; Koga Silva, E.M. Cyanoacrylate Versus Laser in the Treatment of Dentin Hypersensitivity: A Controlled, Randomized, Double-Masked and Non-Inferiority Clinical Trial. J. Periodontol. 2013, 84, 287-294. [CrossRef]

24. Gholami, G.A.; Fekrazad, R.; Esmaiel-Nejad, A.; Kalhori, K.A.M. An evaluation of the occluding effects of Er;Cr:YSGG, Nd:YAG, CO2 and diode lasers on dentinal tubules: A scanning electron microscope in vitro study. Photomed. Laser Surg. 2011, $29,115-121$. [CrossRef]

25. Rizzante, F.A.P.; Maenosono, R.M.; Duarte, M.A.H.; Furuse, A.Y.; Palma-Dibb, R.G.; Ishikiriama, S.K. In Vitro Evaluation of Dentin Hydraulic Conductance After 980 nm Diode Laser Irradiation. J. Periodontol. 2016, 87, 320-326. [CrossRef]

26. Lopes, A.O.; Aranha, A.C.C. Comparative evaluation of the effects of Nd: YAG laser and a desensitizer agent on the treatment of dentin hypersensitivity: A clinical study. Photomed. Laser Surg. 2013, 31, 132-138. [CrossRef]

27. Liu, H.C.; Lin, C.; Lan, W.H. Sealing depth of Nd: YAG laser on human dentinal tubules. J. Endod. 1997, 23, 691-693. [CrossRef]

28. Bal, M.V.; Keskiner, İ.; Sezer, U.; Açıkel, C.; Saygun, I. Comparison of low level laser and arginine-calcium carbonate alone or combination in the treatment of dentin hypersensitivity: A randomized split-mouth clinical study. Photomed. Laser Surg. 2015, 33, 200-205. [CrossRef]

29. Whitters, C.J.; Hall, A.; Creanor, S.L.; Moseley, H.; Gilmour, W.H.; Strang, R.; Saunders, W.P.; Orchardson, R. A clinical study of pulsed Nd: YAG laser-induced pulpal analgesia. J. Dent. 1995, 23, 145-150. [CrossRef]

30. Femiano, F.; Femiano, R.; Femiano, L.; Aresu, G.; Festa, V.M.; Rullo, R.; Perillo, L. Effectiveness of low-level diode laser therapy on pain during cavity preparation on permanent teeth. Am. J. Dent. 2018, 31, 267-271.

31. Jena, A.; Shashirekha, G. Comparison of efficacy of three different desensitizing agents for in-office relief of dentin hypersensitivity: A 4 weeks clinical study. J. Conserv. Dent. 2015, 18, 389-393. [CrossRef] [PubMed]

32. Tabibzadeh, Z.; Fekrazad, R.; Esmaeelnejad, A.; Shadkar, M.M.; Khalili Sadrabad, Z.; Ghojazadeh, M. Effect of combined application of high-and low-intensity lasers on dentin hypersensitivity: A randomized clinical trial. J. Dent. Res. Dent. Clin. Dent. Prospect. 2018, 12, 49-55. [CrossRef] [PubMed] 